\title{
A Review of Electronic Cigarette Use Behaviour Studies*
}

\author{
by \\ Xavier Cahours ${ }^{1}$ and Krishna Prasad ${ }^{2}$ \\ ${ }^{1}$ Imperial Brands, Rue Danton, Fleury-Les-Aubrais, 45404, France \\ ${ }^{2}$ British American Tobacco, Regents Park Road, Southampton, SO15 8TL, United Kingdom
}

\section{SUMMARY}

E-cigarette use has increased markedly in the past five years; however, current data suggest that conventional smokers switching to e-cigarettes may account for much of the recent increase. How individuals use these products has strong implications for nicotine intake and exposure to other potential toxicants. Studies assessing e-cigarette user behaviours, including puff volume and duration, report wide variations across products, settings, and individuals. Understanding the factors that affect puffing topography will be central to standardising protocols for testing aerosol emissions and regulating e-cigarettes. The amount of aerosol generated can be influenced by a number of factors, including product design, vaping topography, and device setting as highlighted in this review. Further work to understand how the combination of these parameters affects the amount of aerosol generated will be central to defining protocols for testing and regulating e-cigarettes. [Beitr. Tabakforsch. Int. 28 (2018) 81-92]

\section{KEYWORDS}

E-cigarette; conventional cigarette; puffing topography; puff volume; puff duration; puff interval; use behaviour.

\section{ZUSAMMENFASSUNG}

Die Nutzung von E-Zigaretten hat in den letzten fünf Jahren deutlich zugenommen. Aktuelle Daten legen allerdings nahe, dass Raucher herkömmlicher Zigaretten, die auf E-Zigaretten umsteigen, für einen Großteil dieser Zunahme verantwortlich sein könnten. Die Art der individuellen Nutzung dieser Produkte hat erhebliche
Auswirkungen auf die Nikotinaufnahme und die Exposition gegenüber anderen potenziellen Giftstoffen. Studien zur Beurteilung des Nutzerverhaltens bei E-Zigaretten, einschließlich Zugvolumen und -dauer, zeigen große Unterschiede je nach Produkt, Geräteeinstellungen und Nutzer. Für die Standardisierung von Protokollen zur Prüfung der Aerosolemissionen und zur Regulierung von E-Zigaretten ist daher das Verständnis der Einflussfaktoren auf die Zugtopographie von zentraler Bedeutung. Wie in diesem Artikel dargelegt, kann die erzeugte Aerosolmenge von einer Reihe von Faktoren, einschließlich des Produktdesigns, der Dampftopographie und der Geräteeinstellungen, beeinflusst werden. Weitere Untersuchungen sind nötig, um zu verstehen, wie die Kombination dieser Parameter die erzeugte Aerosolmenge beeinflusst, da diese von zentraler Bedeutung für die Definition von Protokollen zur Prüfung und Regulierung von E-Zigaretten sein werden. [Beitr. Tabakforsch. Int. 28 (2018) 81-92]

\section{RESUME}

$\mathrm{Au}$ cours des cinq dernières années, les cigarettes électroniques ont nettement gagné en popularité; cependant, les données actuelles laissent à penser qu'une grande partie de ce récent engouement pourrait s'expliquer par la conversion des fumeurs conventionnels à la cigarette électronique. Les modalités d'utilisation de ces produits par les consommateurs ne sont pas sans avoir de profondes implications sur l'absorption de nicotine et sur l'exposition à d'autres composés potentiellement toxiques. Des recherches concernant le comportement des fumeurs de cigarettes électroniques, y compris le volume et la durée de chaque bouffée, mettent en lumière des variations importantes selon les produits, les réglages et les consommateurs. La compréhension des facteurs affectant la topographie de la 
bouffée inhalée s'avèrera essentielle pour la normalisation des protocoles de test des émissions d'aérosols et pour la réglementation des cigarettes électroniques. Le présent article souligne que la quantité d'aérosols générée peut être influencée par un certain nombre de facteurs, notamment la conception du produit, la topographie du vapotage et le réglage de l'appareil. Des études supplémentaires visant à comprendre la façon dont la combinaison de ces paramètres affecte la quantité d'aérosols générés seront indispensables à la définition des protocoles de test et à la réglementation des cigarettes électroniques. [Beitr. Tabakforsch. Int. 28 (2018) 81-92]

\section{INTRODUCTION}

Electronic cigarettes (e-cigarettes, EC) use battery power to heat an element to disperse a solution that usually contains nicotine. Dispersion of the solution leads to the creation of an aerosol that can be inhaled by the user. The heated solution typically contains propylene glycol and/or glycerine, water, nicotine, and flavourings. E-cigarettes do not contain tobacco, do not create smoke, and do not rely on a combustion mechanism. Consequently, they can deliver nicotine to the user with potentially lower exposure to the harmful constituents that are produced by the combustion of conventional tobacco cigarettes.

There is substantial heterogeneity among the different types of e-cigarettes, and the speed with which they are evolving makes them difficult to categorize. There are many designs, including cigarette-shaped formats with various lengths and circumferences, but also devices with completely different shapes. Regardless of format, most devices are available as one-, two- or three-piece products. Two-piece products consist of a battery and a cartomizer (comprising a cartridge and atomizer); in three-piece products, the cartridge and atomizer are separated. The devices may be disposable or reusable, with rechargeable batteries and refillable cartridges or tanks. Some e-cigarettes can be configured by the user, by selection of a liquid with a preferred flavour or nicotine concentration, and/or a device modification such as increased heating, higher battery power, variable voltage controllers, or larger e-liquid tanks. Using an e-cigarette is sometimes known as 'vaping' and the user as a 'vaper' (analogous to 'smoking' and 'smoker', respectively).

Since their introduction to the market in 2004, global usage of e-cigarettes has risen exponentially. Changes in consumer preference and a desire for a healthier lifestyle are driving this shift, with mature tobacco markets leading the way. The United States dominate the market for vapour products, accounting for nearly half of all global sales, followed by the United Kingdom, Italy, Poland, and France. The e-cigarette has been rapidly gaining ground on conventional cigarettes, and could surpass consumption of conventional cigarettes within the next decade, according to some prediction analyses (1). The competitive challenge posed to cigarettes by vapour products eased slightly in 2016 , as the category's growth all but halved to $32 \%$, representing a total global value of US \$ 8 billion (2). In category terms, the well-documented shift from "cig-a-likes" to tank systems continued in 2015. Excluding the U.S. market, where the split is much closer to 50/50, tank systems accounted for $85 \%$ of global e-cigarette use with "cig-a-likes" accounting for only $15 \%$.

Information on vaping behaviour can provide understanding into puffing topography, potential nicotine uptake, as well as variability or unique patterns of usage between different e-cigarette types and users. In addition, topography data are needed to understand baseline characteristics related to e-cigarette use, which can then be used to establish standardized vaping machine protocols. Such standards would be valuable to industries and regulators working with or studying these products.

E-cigarette use is significantly different compared to smoking, however, at this time, very limited information is available on topography and subjective effects of e-cigarettes. One of current objectives of the CORESTA ${ }^{1}$ Product Use Behaviour Sub-Group is to identify gaps in available information and suggest suitable work to fill the gaps. Before proposing studies, it is necessary to review all relevant e-cigarette use-behaviour studies published with a view to produce a comprehensive knowledge base of all relevant work in the field. Therefore, the aim of this review is to examine the topography of e-cigarette use, including the instrumentation and methods being developed to measure parameters such as puff duration, interval, and volume, to build a picture of puffing behaviour among e-cigarette users. Characterising such behaviour is important in standardising protocols for testing and regulating e-cigarettes.

\section{METHOD}

Articles published in peer-reviewed journals were retrieved from PubMed (http://www.ncbi.nlm.nih.gov/pubmed/) available as of December 2017 using combinations of the following keywords: "electronic cigarette" or "e-cigarette" or "ENDS" or "electronic nicotine delivery system" or "electronic nicotine delivery device" and "topography" or "smoking behaviour" or "vaping behaviour". To be sure to have all relevant information regarding vaping behaviour, we combined to the initial searches the following search terms prevalence/consumption/pattern/reason for use. In addition, references of the retrieved articles were examined to identify further relevant articles, with particular attention paid to non-peer reviewed reports and conference presentations. Unpublished results obtained through personal communications were also reviewed. After removing duplicates, recommendations, expert statements, reviews and technical reports this search identified 169 records. All records were read by two reviewers using a protocol with clearly identified inclusion and exclusion criteria. Papers on smoking cessation, consumption, prevalence, attitudes, and beliefs without any information on vaping behaviour and/or topography were excluded. Papers were deemed relevant ( $\mathrm{n}=28$, publications and congress presentations) to this review if they provided key information on vaping behaviour and topography. Only peer-reviewed publications that presented original data were kept for the Table 1.

\footnotetext{
${ }^{1}$ CORESTA (Cooperation Centre for Scientific Research Relative to Tobacco) is a non-profit association, the purpose being to promote international cooperation in scientific research relative to tobacco and its derived products. https://www.coresta.org.
} 


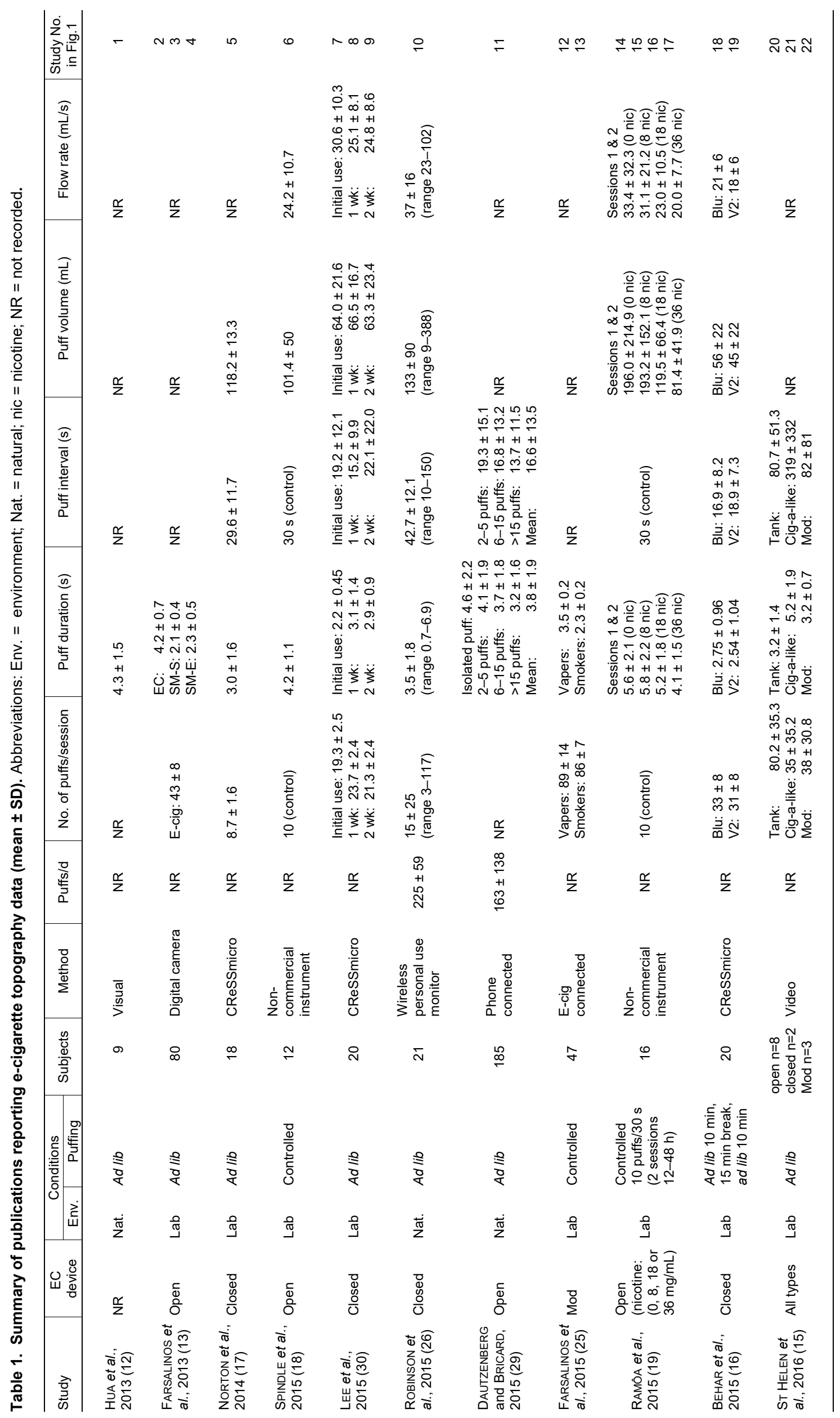




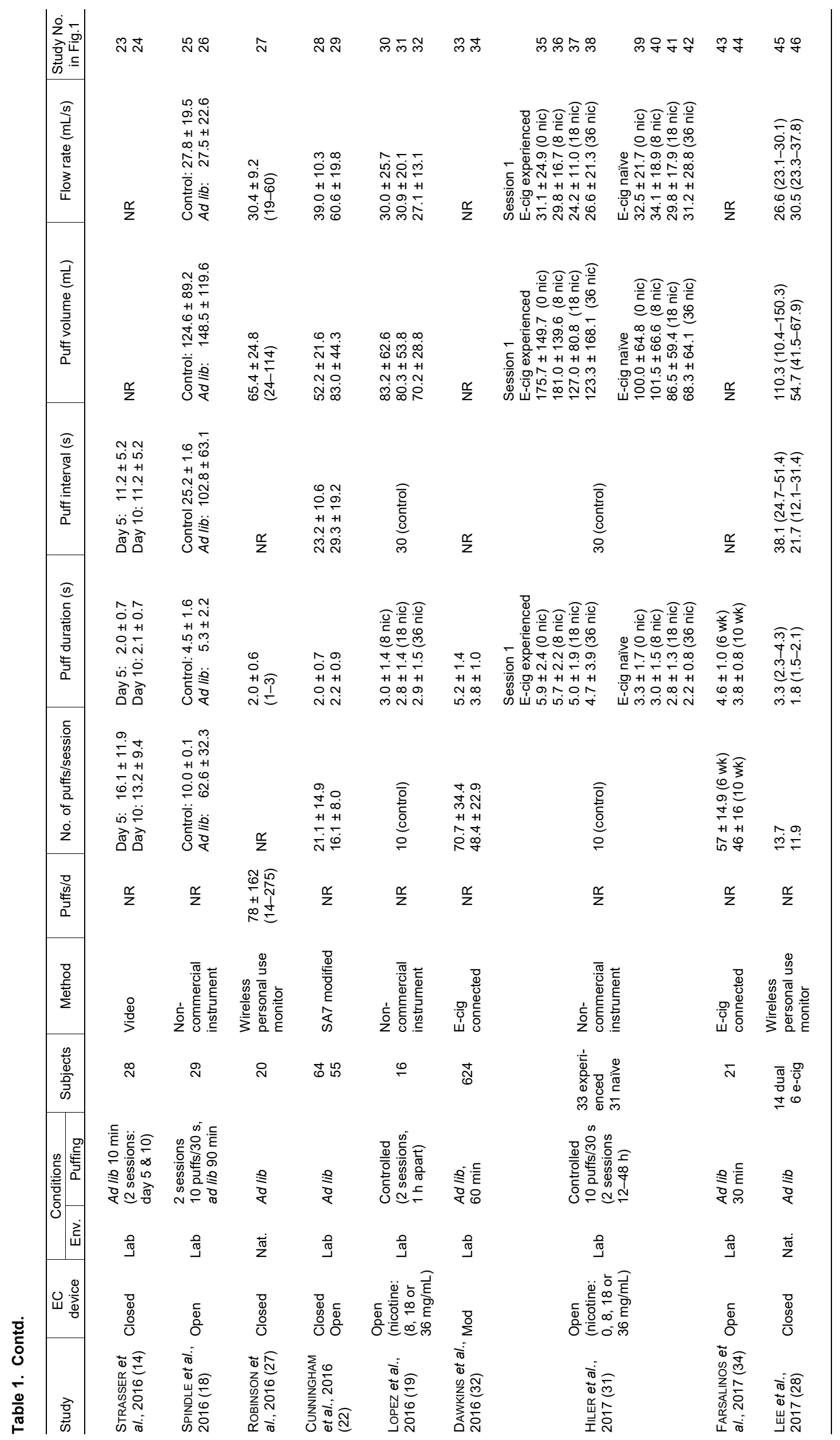




\section{E-cigarette topography}

E-cigarette topography data are needed both to characterize an individual's risk of exposure to nicotine and other potential toxicants, and to establish baseline parameters for regulatory protocols. Conventional cigarette topography and smoking behaviour have been widely studied (3-5) and the methods are continuing to evolve $(6,7)$. Data collected by these approaches can be used to establish the validity of standardized machine-smoking protocols such as ISO intense and non-intense $(8,9)$ that are essential for regulatory assessments of aerosol emissions.

By contrast, e-cigarette topography studies are still in their early days. As yet, there is no standardized protocol for testing emissions from e-cigarettes, although, towards this end, CORESTA set up a Task Force Study in 2013. At the beginning, there was extensive discussion regarding puffing parameters. The primary concerns discussed related to insufficient product trigger (time/heat), insufficient overlap between the machine (bell) profile and/or machine duration and the start and end times for some products' puff time. Puff durations of $5 \mathrm{~s}$ or longer were recommended against as being too long from some products. In order to prepare for a future proficiency study, the Task Force (TF) commissioned a study to determine the effect of puff profile on the yield of the major constituents in e-cigarette aerosols in order to recommend puffing parameters for aerosol collection. The study involved comparison of puffing parameters combinations including 55-mL and 70-mL puff volumes of $3 \mathrm{~s}$ or $4 \mathrm{~s}$ duration. Based on TF discussion and on the results of this study, vaping parameters of $55 \mathrm{~mL}$ volume taken over $3 \mathrm{~s}$ every $30 \mathrm{~s}$ using a square profile shape were recommended for adoption as an interim standard vaping regime for e-cigarette testing (10). In 2015, the CORESTA published a recommended method for machine-smoking e-cigarettes (11).

It is interesting to see how the emerging topography data from e-cigarette users compare to the CORESTA recommended machine-puffing parameters of $3 \mathrm{~s}, 55 \mathrm{~mL}$ and $30 \mathrm{~s}$ for puff volume, puff duration, and inter-puff interval, respectively.

\section{Methods for assessing the topography of e-cigarette use}

\section{- Video recording}

Initial behaviour studies used video recordings of users to report some topography parameters. In 2011, for example, HUA et al. (12) analysed puff and exhalation duration for 64 individuals using e-cigarettes and 9 individuals smoking conventional cigarettes in YouTube videos. For e-cigarette users, puff duration was defined as the interval between the LED light switching on and the first exhalation. They found that mean puff duration varied across brands from $3.6 \mathrm{~s}$ to $5.8 \mathrm{~s}$.

FARSALINOS et al. (13) compared patterns of product use among 45 experienced e-cigarette users and 35 experienced smokers by using a camera to record the participants. Puff, inhalation, and exhalation durations were measured by frame-by-frame analysis. Significant differences were observed between the e-cigarette group and the smokers group in their patterns of puffing. Puff duration, for exam- ple, was twofold higher in the e-cigarette group than in the smoker group. Inhalation duration, by contrast, was shorter in the e-cigarette group.

More recently, STRASSER et al. (14) assessed how two types of e-cigarette are used relative to conventional cigarettes among US current smokers who switched to e-cigarettes. Although computerized topography measurement systems were available, they used video recording for topography measurements, arguing that different mouthpieces would be required for each product (cigarettes, first-generation "cig-a-likes", tanks) and might affect use patterns and complicate comparisons across the study products. Their study found significant differences in user topography (puff duration and inter-puff interval) between e-cigarettes and conventional cigarettes $(\mathrm{p}<0.05)$.

The video-recording method has also been used to characterize e-cigarette topography among 13 adult e-cigarette users (15). The authors argued that, although handheld devices designed for use with conventional cigarettes had been validated for "cig-a-likes", they were not designed for use with tank-type products or rebuildable atomizer models. As in previous studies, e-cigarette topography was found to differ from smoking topography. Although video analyses can be useful, they are limited in the number of parameters that can be obtained; for example, puff velocity and volume cannot be measured from the recordings. Moreover, a high observer bias may occur when determining puff duration times because there is subjective visual interpretation. Therefore, electronic devices that can quantitatively measure the topography parameters of e-cigarette are being developed and validated.

\section{- Computerized topography measurement systems}

In a study to quantify nicotine intake among e-cigarette users, BEHAR et al. (16) evaluated e-cigarette topography with a Clinical Research Support System (CReSS) Pocket, a handheld device developed to measure smoking parameters (e.g., puff duration and count, inter-puff interval, total volume, flow rate and peak flow rate) among conventional cigarette smokers. Participants consumed two types of e-cigarette ad libitum for $10 \mathrm{~min}$ through the CreSS Pocket device. The CreSS Pocket was validated for use with e-cigarettes in terms of pressure drop, flow rate, and volume; however, it was found to underestimate the total e-cigarette puff counts. The CReSS Pocket is pre-set by the manufacturer to stop recording topography data after a user reaches 43 puffs, and $26 \%$ of the users in the study exceeded this puff number during the 10 minute ad libitum use. It also underestimated the average puff duration because the large puffs taken by some participants drew fluid into the device, and so these data were excluded from the analysis. By excluding those topography profiles, the study did not capture the true e-cigarette topography demographic. NORTON et al. (17) used a CreSSmicro ${ }^{\mathrm{TM}}$ portable smoking topography device, coupled with a specialized adapter from the manufacturer, to record the topography of e-cigarette use. They successfully measured puff number, volume and puff duration, inter-puff interval, and average flow, although data were lost for some participants due to equipment failure.

SPINDLE et al. (18) have compared nicotine delivery, heart 
rate and the subjective effects of experienced e-cigarette users consuming their preferred e-cigarette with and without a mouthpiece-based topography recording device. Puff volume, duration, number, interval between puffs, and flow rate were recorded by an instrument similar to conventional cigarette topography instruments that was developed specifically for e-cigarettes at the American University of Beirut. Preliminary findings among 13 e-cigarette users who completed the study showed that use of the mouthpiece-based topography measurement device had no effect on nicotine delivery, heart rate or subjective effects of e-cigarette use on withdrawal (18). This finding was further supported by a study among 29 e-cigarette users (18), which found that puffing topography among e-cigarette users differed significantly between directed use and ad libitum use, suggesting that research based on ad libitum use of e-cigarettes would provide better understanding of e-cigarette behaviour and toxicant dose.

The same e-cigarette topography instrument has also been successfully used in two other studies to examine the relationship between liquid nicotine concentration, plasma nicotine concentration, and puffing behaviour among experienced e-cigarette users with topography findings consistent with previous results as discussed earlier (19).

At the 2014 CORESTA congress, CUNNINGHAM et al. (20) described a device for measuring the puffing topography of e-cigarette users. The Smoking Analyser Number 7 (SA7), developed by British American Tobacco and C-Matic Systems Ltd., was modified to overcome issues caused by the condensation of humectants from the e-cigarette in the topography head. The performance of the modified device was validated for disposable, cartomizer-based, and modular e-cigarettes, with a puff volume accuracy comparable to the $6.0 \%$-level observed for combustible cigarette aerosol measurements. The modified SA7 has been used to determine the puffing topography of regular users of e-cigarettes using either cartomiser or closed-modular products (21). In 2016, CUNNINGHAM et al. (22) further tested the modified SA7 using air and e-cigarette aerosol under multiple regimes. Puffing topography was measured among 60 experienced e-cigarette users who were given rechargeable "cig-a-like" or larger button-activated e-cigarettes to use ad libitum in two sessions. Under all regimes, air puff volumes were within $1 \mathrm{~mL}$ of the target and aerosol volumes within $5 \mathrm{~mL}$ for all e-cigarette types, serving to validate the topography instrument.

CONNELL et al. (23) examined use of the SODIM Smoking Puff Analyser Mobile (SPA-M) to measure the puff duration, volume, and profile of an e-cigarette prototype. The validation process included verifying the calibration, accuracy, precision and robustness of the SPA-M device against known instrumentation and calibration artefacts. The performance of the SPA-M under standard settings was not acceptable for use with the e-cigarette. Therefore, the SPA-M factory default values entered into the SPA-M calibration software needed to be modified for e-cigarettes using a K-coefficient to compensate for differences in e-cigarette aerosol composition and temperature influences. Using this K-coefficient, the SPA-M device met validation acceptance criteria for the following experiments: calibration, accuracy, precision, robustness, and system suitability.
The same group conducted a subsequent study to determine the suitability and dependability of the validated SPA-M for puff topography measurement in a clinical setting (24). Puff topography was measured among exclusive conventional cigarette smokers $(\mathrm{n}=13)$ and exclusive e-cigarette users $(\mathrm{n}=10)$ under conditions of 7-hour ad libitum use. It was concluded that the SPA-M is suitable for measuring the topography of the e-cigarettes used in the study.

\section{- Connected e-cigarettes and wireless monitors}

New "connected e-cigarettes" that automatically record all presses of the e-cigarette activation switch have been recently launched. These devices can record the time of activation of each puff, and store the puff number, duration and time of each puff in their internal memory. FARSALINOS et al. (25) compared nicotine absorption between experienced e-cigarette users and naïve users (smokers) using connected e-cigarettes during an ad libitum laboratory session of $60 \mathrm{~min}$. The accuracy of the recorded data was validated in a pilot study, in which 5 experienced users were asked to use the device for 30 puffs. The puff number was manually counted and compared with the recorded number of puffs. Additionally, the accuracy of puff duration recording was validated by video recording 5 puffs per participant and comparing the durations between video recording and device record.

Puff topography data have been mostly obtained in a laboratory environment over a limited session time. Only a few researchers have assessed the usage patterns of e-cigarette users in their natural environment. In a US study, ROBINSON et al. (26) quantified variation in the puffing behaviour of a given user over the course of a day, as well as variations among different users. Puffing topography was measured with a wireless personal use monitor (wPUM), which was designed, built and tested at the Rochester Institute of Technology. For each e-cigarette session, the wPUM recorded the number of puffs, mean puff duration, mean puff flow rate, mean puff volume, and cumulative puff volume. This was the first study to present topography and frequency of use data for 22 e-cigarette users in their natural environment over a 24-hour period. In a follow-up study, RoBINSON et al. (27) used the same device to monitor topography among young adults using e-cigarettes for one week in their natural environment. With the exception of one e-cigarette user, the device functioned as expected with no loss of data. More recently, LEE et al. (28) also used a hand-held wireless device to monitor e-cigarette usage among 20 young adults (18-25 years) in their everyday lives over 1 week.

In a French study aimed at characterizing e-cigarette usage in a real-world setting, DAUTZENBERG and BRICARD (29) reported an analysis of 1 million puffs generated by 185 users consuming connected e-cigarettes, which recorded all puffs with a duration greater than $0.5 \mathrm{~s}$. The 1 million puffs were produced from the devices in 116 days, and the collected data extensively documented usage patterns (including mean puff number and puff duration) with large variations observed among different users and on different days for the same user (usage was greater at the weekend). Although the study analysed accurate usage data recorded directly on the devices, no information was collected on 


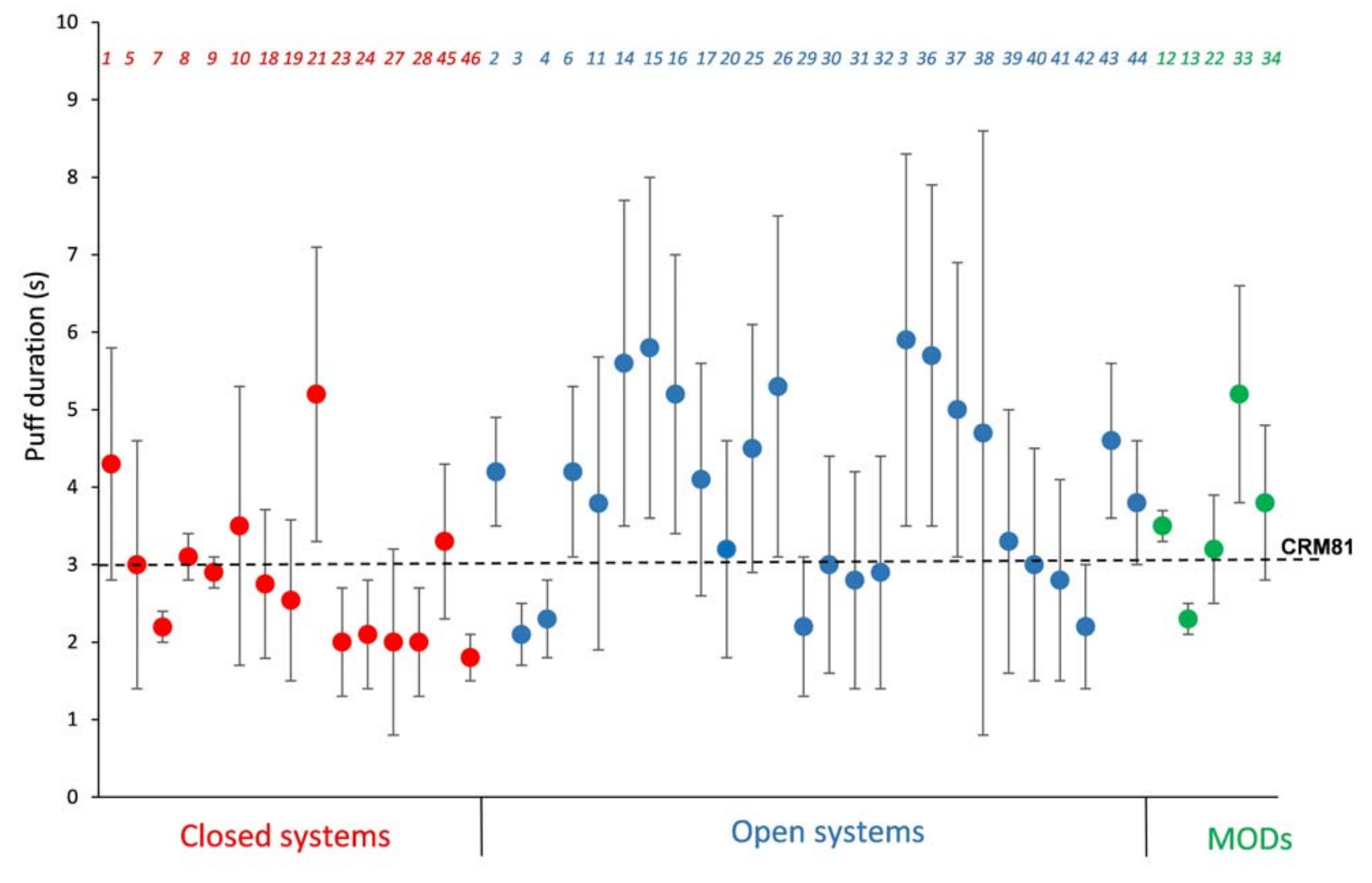

Figure 1. Mean puff durations reported in the publications cited in Table 1 (No. 1-46). The dotted line indicates the puff duration (i.e., $3 \mathrm{~s}$ ) recommended in CORESTA Method No. 81 (9). Across all studies, regardless of population or product type, the mean puff duration ranged from $2 \mathrm{~s}$ to $5.8 \mathrm{~s}$ (grand mean $3.4 \mathrm{~s}$ ). By product type, the grand mean puff duration was $2.9 \mathrm{~s}$ for "cig-a-likes", $4.0 \mathrm{~s}$ for open tank systems, and $3.0 \mathrm{~s}$ for MOD systems. High variability was observed in some studies, leading to large variability around the grand mean.

user age, sex, or concomitant use of tobacco or other e-cigarettes. The authors concluded that further studies with other devices and characterization of the users are needed before definitive conclusions can be drawn on patterns of e-cigarette usage.

In summary, various research groups have conducted multiple studies to better understand e-cigarette topo graphy, but there remain gaps in the research that need to be understood and addressed in order to progress in future studies, especially in the investigation of e-cigarette topography in the natural environment.

\section{E-cigarette topography data}

In the past five years, a number of studies have reported e-cigarette topography data (Table 1). The characteristics of the study subjects, types of e-cigarette used, and measurement methods vary considerably, resulting in a wide range of reported values that deviate considerably from the machine-puffing parameters recommended by CORESTA (11). As can be seen in Table 1 and Figures 1-3, the reported mean puff duration of e-cigarette use ranges from $2 \mathrm{~s}$ to $5.8 \mathrm{~s}$, the inter-puff interval from $11.2 \mathrm{~s}$ to a massive $319 \mathrm{~s}$, and puff volume from $45 \mathrm{~mL}$ to $193 \mathrm{~mL}$. Below, we summarize the main findings from these studies by the aims of the original investigations.

\section{- Effect of experienced versus naïve e-cigarette use}

One clear finding is that there is a substantial difference in topography patterns between experienced and naïve EC users. Using a video-recording approach to measure topography among 80 volunteers, FARSALINOS et al. (13) found that mean puff duration was significantly higher among regular e-cigarette users (4.2 s) than among conventional smokers either smoking conventional cigarettes $(2.1 \mathrm{~s})$ or using e-cigarettes (2.3 s). FARSALINOS et al. (13) suggested that e-cigarette users take a longer puff owing to the delay between activation of the e-cigarette and the sufficient production of aerosol. By contrast, smokers inhale from a lit cigarette and can draw harder to increase the puff volume and thus nicotine-mediated subjective effects. FARSALINOS et al. (25) also evaluated puffing topography among smokers with no experience of e-cigarette use $(n=23)$ and experienced users $(n=24)$ using a new-generation connected cigarette. Both groups took a similar number of puffs within the 65-minute laboratory session. Similar to their previous study, however, mean puff duration was $3.5 \mathrm{~s}$ (range 1.8-6.2 s) among experienced users and $2.3 \mathrm{~s}$ (range 0.9-4.3 s) among smokers $(\mathrm{p}<0.001)$.

Using the CreSSmicro device, LEE et al. (30) assessed changes in puffing behaviour among adult smokers who were naïve to e-cigarettes and switched from conventional 


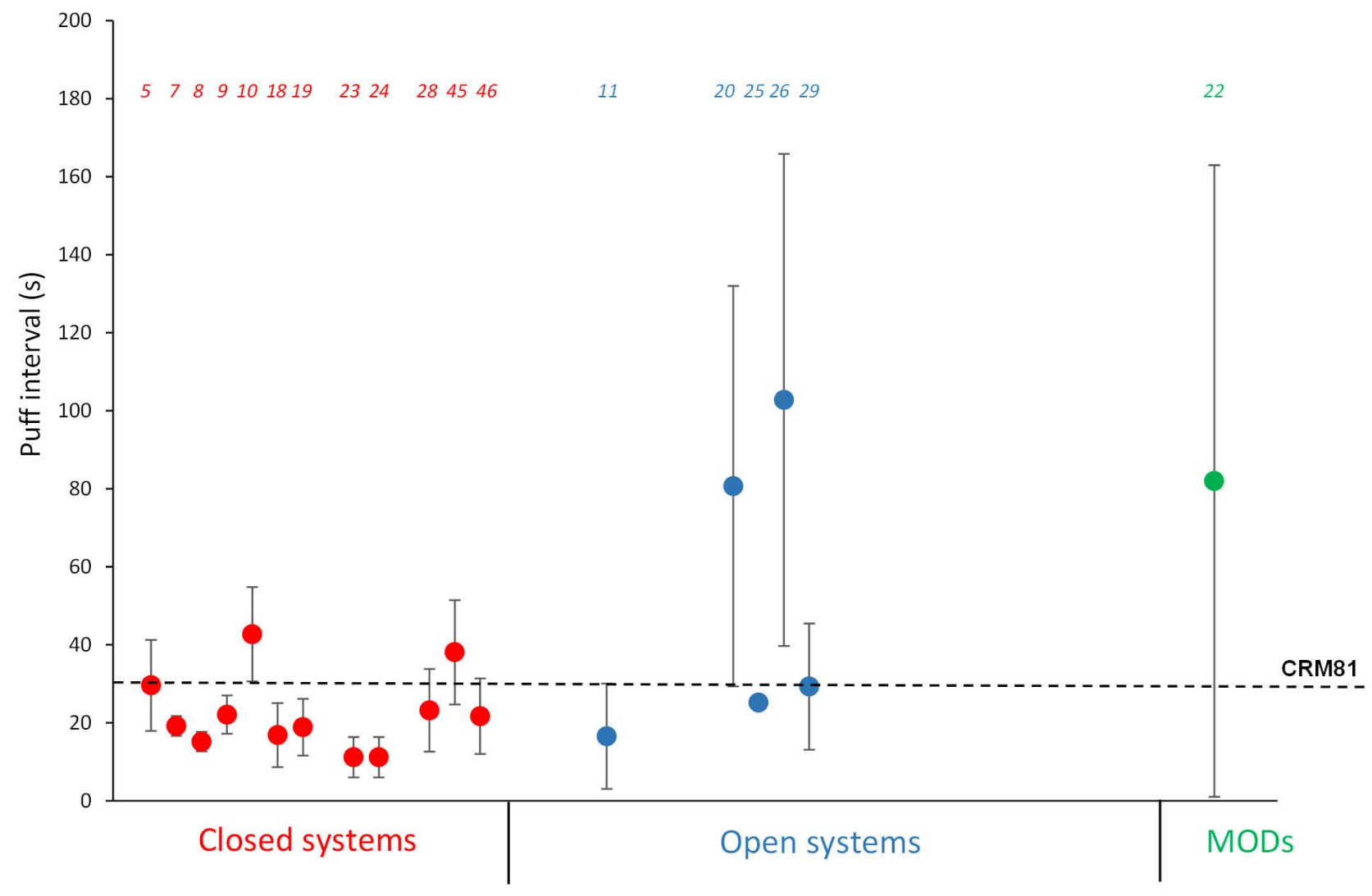

Figure 2. Mean inter-puff interval reported in the publications cited in Table 1 (No. 5-11, 18-20, 22-26, 28, 29). Note that values from study 21 were not included owing to the high mean \pm SD $(319 \pm 332 \mathrm{~s})(13)$. The grand mean value for the "cig-a-likes" shown (21.0 s) is close to the puff interval duration ( $30 \mathrm{~s}$ ) recommended in CORESTA Method No. 81 (9); if the value from study 21 is included, however, the grand mean value for "cig-a-likes" rises to $48.1 \mathrm{~s}$. Only five studies have reported the puff interval for open tank systems and show high inter-study variability: $16.6 \mathrm{~s}, 25.2 \mathrm{~s}, 29.3 \mathrm{~s}, 80.7 \mathrm{~s}$, and $102.8 \mathrm{~s}$.

to e-cigarettes for 2 weeks. After 1 week of e-cigarette use, smokers showed a significant increase in puff duration from $2.2 \mathrm{~s}$ to $3.1 \mathrm{~s}(\mathrm{p}<0.05)$. Simultaneously, the mean puff flow rate decreased from 30.6 to $25.1 \mathrm{~mL} / \mathrm{s}(\mathrm{p}<0.05)$. No further changes in puffing behaviour were observed in the second week of the study, which suggests that smokers took only a few days to adjust to the new product. The authors concluded that the smokers most probably changed their puffing behavior to compensate for the less-efficient delivery of nicotine from e-cigarettes.

HILER et al. (31) also compared topography between experienced e-cigarette users and smokers with no experience in e-cigarette use. Again, their results confirmed that experienced e-cigarette users take significantly longer $(5.6 \mathrm{~s} v \mathrm{~s}$ $2.9 \mathrm{~s})$ and larger $(161.5 \mathrm{~mL} / \mathrm{s}$ vs $94.0 \mathrm{~mL} / \mathrm{s})$ puffs relative to e-cigarette-naïve smokers.

\section{- Effect of type/brand of e-cigarette}

BEHAR et al. (16) evaluated e-cigarette topography parameters among 20 volunteers using two "cig-a-like" brands, and calculated nicotine intake from the cartomizer fluid consumed and puff count. Topography parameters were recorded by the CreSS Pocket while the participants used each e-cigarette ad libitum in two laboratory sessions. Apart from total puff count, all topography parameters differed significantly, which might be related to e-cigarette performance and differences in the efficiency of nicotine delivery between the two brands.

Using video recordings, ST HELEN et al. (15) characterized use and nicotine intake during a 90-minute period of $a d$ libitum access among experienced e-cigarette users consuming their usual e-cigarettes including "cig-a-likes" with disposable or rechargeable cartridges $(\mathrm{n}=2)$ or tanks $(\mathrm{n}=8)$, and customizable advanced personal vaporizers or "MODs" $(\mathrm{n}=3)$. While they did not compare the topography of the different product types, computing the average puff duration for each product type shows differences, especially between the "cig-a-likes" (5.2 s) and tanks $(3.2 \mathrm{~s})$ or MODs $(3.2 \mathrm{~s})$; however, there were only two "cig-a-like" users consuming two different products with vastly different usage patterns (e.g., puff count, 60 and 10; puff duration, 6.6 and $3.9 \mathrm{~s}$ ). The variation in puff number among the tank e-cigarette users was also wide (ranging from 37 to 121 puffs in a 90-minute session). These results show that further research on product types is warranted in different settings among different volunteer groups.

CUNNINGHAM et al. (22) reported ad libitum puffing topography of 60 e-cigarette users consuming rechargeable "cig-a-like" and larger button-activated e-cigarettes. Mean puff durations ( $2.0 \mathrm{~s}$ and $2.2 \mathrm{~s}$, respectively) were similar with both types of e-cigarette, but mean puff volumes $(52.2 \mathrm{~mL}$ and $83.0 \mathrm{~mL}$, respectively) and mean inter-puff intervals (23.2 $\mathrm{s}$ and $29.3 \mathrm{~s}$, respectively) differed signifi- 


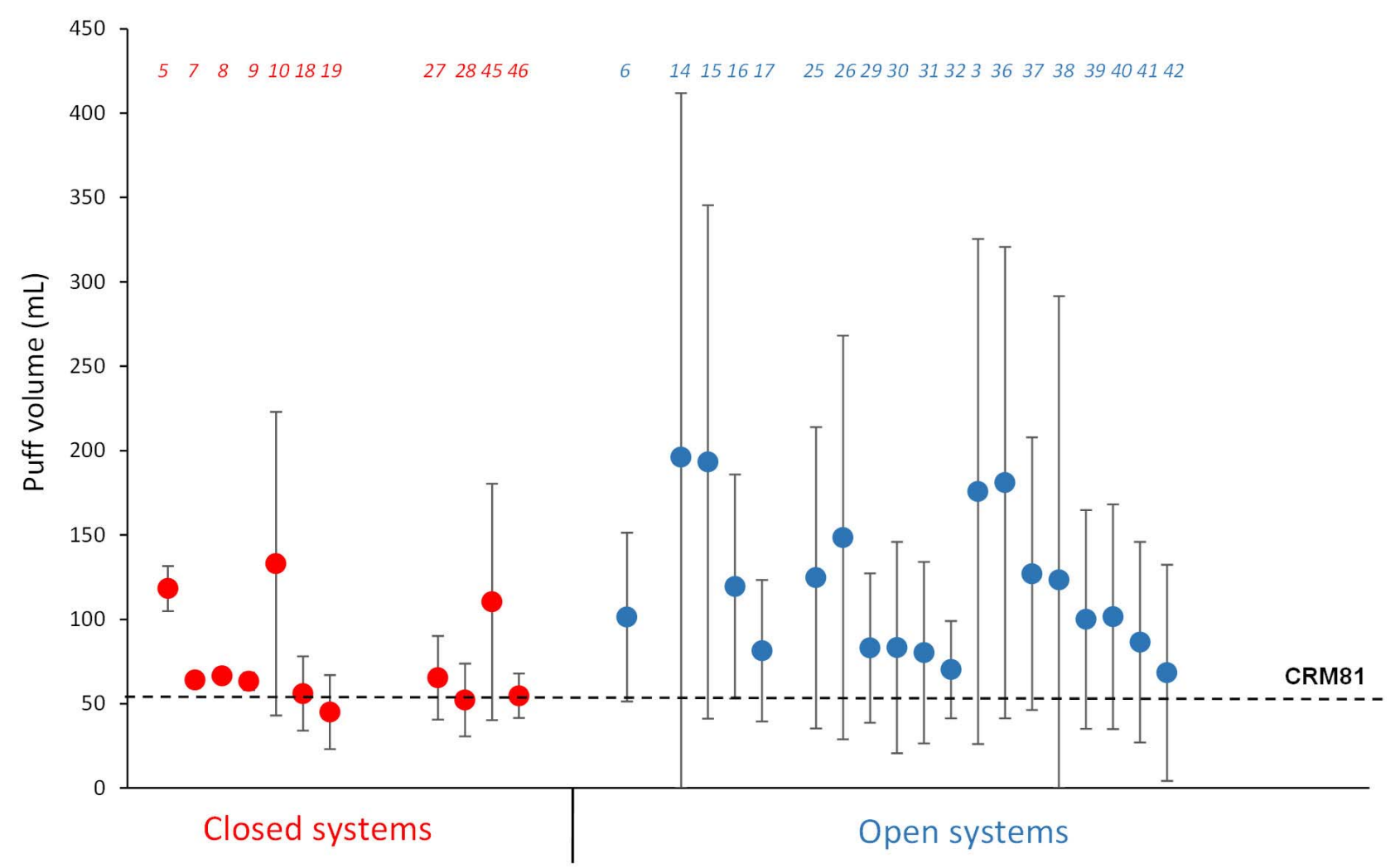

Figure 3. Mean puff volume reported in the publications cited in Table 1 (No. 5-10, 14-19, 25-32, 35-42, 45, 46). The grand mean puff volume is $94.7 \mathrm{~mL}$ (range $45-193 \mathrm{~mL}$ ). By product type, the grand means are $73.3 \mathrm{~mL}$ for "cig-a-likes" and $121.7 \mathrm{~mL}$ for open tank systems. In some studies, the intra-study variability was very high, for example, $196 \pm 214.9 \mathrm{~mL}$ for study 14 (19), and $123.3 \pm 168.8 \mathrm{~mL}$ for study 38 (31). At present, there is no information on puff volume for MOD systems.

cantly. The data clearly showed that product characteristics influence puffing topography, and therefore the results obtained from a given e-cigarette might not be generalizable to other products. As concluded by the authors, it will be important to determine all of the factors that affect puffing parameters in order to develop standardized testing protocols for e-cigarette emissions.

Type of e-cigarette is likely to have a big impact on topography parameters. For example, among the data published so far (Table 1), puff durations seem to be higher for open systems (3-4 s) than for closed systems (2-3 s). However, differences in the study populations and protocols do not facilitate a true comparison.

\section{- Effect of nicotine concentration and battery power}

A few studies have investigated whether the nicotine concentration of the e-cigarette or e-liquid affects puffing topography. RAMÔA et al. (18) studied the effect of liquid nicotine concentration on the puffing topography of experienced e-cigarette users. The reported mean puff duration was $5.5 \mathrm{~s}$ for $0 \mathrm{mg} / \mathrm{mL}, 5.5 \mathrm{~s}$ for $8 \mathrm{mg} / \mathrm{mL}, 4.97 \mathrm{~s}$ for $18 \mathrm{mg} / \mathrm{mL}$ and $3.98 \mathrm{~s}$ for $36 \mathrm{mg} / \mathrm{mL}$, with a significant difference between 0 and $36 \mathrm{mg} / \mathrm{mL}$.

Recently, DAWKINS et al. (32) examined the effect of high and low nicotine e-liquid concentrations on puffing topography among 11 experienced males over two ad libitum laboratory sessions. E-liquid consumption and puff number were higher and puff duration was longer for low nicotine e-cigarettes (all $\mathrm{p}<0.01$ ). The authors concluded that e-cigarette users changed their smoking behaviour with nicotine strength, doubling their consumption of e-liquid for low-nicotine products.

In their study of experienced and inexperienced e-cigarette users, HILER et al. (31) also found that puffing topography was correlated with liquid nicotine concentration. For example, the mean puff duration was $5.9 \mathrm{~s}$ for $0 \mathrm{mg} / \mathrm{mL}$ and $4.7 \mathrm{~s}$ for $36 \mathrm{mg} / \mathrm{mL}$ of nicotine among experienced e-cigarette users, and $3.3 \pm 1.7 \mathrm{~s}$ for $0 \mathrm{mg} / \mathrm{mL}$ and $2.2 \mathrm{~s}$ for $36 \mathrm{mg} / \mathrm{mL}$ of nicotine among naïve e-cigarette users. Puff volume similarly decreased with increasing nicotine concentration (Table 1). Thus, irrespective of e-cigarette experience, users adjusted their behaviour according to the amount of nicotine in the e-liquid.

The type of e-cigarette used also influences nicotine delivery. For example, plasma nicotine levels were found to be higher in users of third-generation e-cigarettes than in users of second-generation devices (33). This difference is likely to be due to design characteristics and the power delivery potential of more recent e-cigarette devices. Clearly, the topography parameters of e-cigarette use change with the nicotine concentration of the e-liquid. At present, however, it is not clear whether users alter their puff topography to titrate nicotine delivery or to avoid secondary effects of the nicotine (e.g., headache, scratchiness, bad taste, and so on). 
Recently, FARSALINOS et al. (34) evaluated changes in puffing topography and liquid consumption among e-cigarette users when using different power settings $(6 \mathrm{~W}$ and $10 \mathrm{~W}$ ) for the battery in third-generation connected e-cigarettes. Users were allowed to use the e-cigarette ad libitum but were blind to the power setting. Battery power was found to affect puffing topography, with users taking 19\% fewer and $17 \%$ shorter puffs on the high-power setting.

\section{- Effect of environment on recorded parameters}

As mentioned above, SPINDLE et al. $(35,36)$ showed that a mouthpiece-based topography recording device does not affect the study outcomes, even under ad libitum conditions of e-cigarette use. However, they found that protocols based on controlled conditions (e.g., fixed number of puffs and inter-puff interval) may alter other topography outcomes (e.g., puff duration) that are not controlled experimentally. For example, e-cigarette users had a longer mean puff duration $(5.3 \mathrm{~s})$ in the ad libitum condition than in the controlled puff number condition $(4.5 \mathrm{~s})$. In addition, there were significant differences in mean puff volume (controlled, $124.6 \mathrm{~mL}$; ad-lib, $148.5 \mathrm{~mL}$ ) and inter-puff interval (controlled, $25.2 \mathrm{~s}$; ad-lib, $102.8 \mathrm{~s}$ ).

Recently, there has been a trend towards recording topography during the course of normal use by e-cigarette consumers, facilitated by the development of the connected e-cigarettes and wireless monitors described above. RoBINSON et al. $(26,27)$ have conducted two studies of e-cigarette users in their natural environment. In their first study, the topography of 21 subjects using "cig-a-likes" over the course of a normal day ( 24 hours) was recorded by using a wireless monitor. A total of 295 sessions and 4723 puffs were recorded with a mean total number of 225 puffs per day (range 24 to $>1000$ puffs/day). The mean puff duration was $3.5 \mathrm{~s}(0.7-6.9 \mathrm{~s})$, and the mean puff volume was $133 \mathrm{~mL}(29-388 \mathrm{~mL})$. The standard deviations observed in the puff topography characteristics (see Table 1) indicate that individual puffing patterns vary significantly over the course of a 24-hour period.

To improve understanding of both intra-user variability (variation in behaviour for a given user) and inter-user variability (variation in behaviour among users in a given cohort), RoBINSON et al. $(26,27)$ followed up the study with a week-long evaluation of 20 young adults using their normal e-cigarettes (all "cig-a-like") in their natural environment. Significant intra-participant variability was observed for puff duration and puff volume, with some users showing a wide spread in topography parameters over the week and others a relatively low spread. For example, user-specific standard deviations ranged from 0.8 to $4.1 \mathrm{~s}$ for puff duration, 6 to $23 \mathrm{~mL} / \mathrm{s}$ for puff flow rate, and 24 to $164 \mathrm{~mL}$ for puff volume.

Based on the outcomes of that study, the same research group suggested that prior experience of smoking cigarettes might result in distinct e-cigarette puffing topography due to factors such as learned inhalation behaviour or nicotine dependence. In a subsequent study, therefore, they compared topography between 14 established cigarette smokers who also used e-cigarettes and 6 non-cigarette smokers using closed system e-cigarettes in their natural environment over 1 week (28). As found in the studies summarized in the section "Effect of experienced versus naïve e-cigarette use", established cigarette smokers used e-cigarettes for longer puff durations (3.3 vs $1.8 \mathrm{~s}$ ), had longer interpuff intervals (38.1 vs 21.7s), and had larger puff volumes (110.3 vs $54.7 \mathrm{~mL}$ ) as compared with users who did not smoke cigarettes.

As mentioned above, DAUTZENBERG and BRICARD (29) reported the topography analysis of 1 million puffs generated by 185 users of connected e-cigarettes in their natural environment. Their study provides a substantial body of topography data on individual usage and variability, including how usage changes over the course of a day and between week days and weekends, as well standard data on puff duration, inter-puff interval and daily puff count. Notably, puff duration was found to increase during the first months of use of the connected e-cigarette from $3.40 \mathrm{~s}$ on day 1 of use to $4.11 \mathrm{~s}$ on day 60 . In addition, puff duration was found to be longer when the puff was isolated (i.e., without another puff $60 \mathrm{~s}$ before or $60 \mathrm{~s}$ after), although only a minority of puffs were "isolated puffs". As the results were based on one brand of connected e-cigarette sold in France with no demographic information about the users, the study findings have limitations in terms of generalization. Other products such as MODs have different electric power settings, different atomizers, and different chambers volumes, and so are likely to have different puffing patterns.

\section{CONCLUSION}

Although awareness and use of e-cigarettes is continuing to rise globally, current data suggest that much of the increase is due to conventional smokers using e-cigarettes as a means to either cut down cigarette consumption or quit smoking altogether. Health authorities have concluded that e-cigarettes pose fewer health risks than conventional cigarettes (37); nevertheless, how individuals use these products has strong implications for nicotine intake and exposure to other potential toxicants.

As seen in this review, studies assessing user behaviours report wide variations in puff volume, puff duration and inter-puff interval across products, settings, and individuals. The question of which puffing regime would provide better topography characterisation in terms of regulation is difficult because no single regime is likely to represent true human behaviour or produce emissions tightly linked to human exposure or risk, either for individual smokers or for population-level differences between brands.

CORESTA Recommended Method No. 81 lays out the essential requirements necessary to generate and collect e-cigarette aerosol for analytical testing purposes (11); however, the recommended parameters do not reflect intense use. The amount of aerosol generated can be influenced by a number of factors, including product design, vaping topography, and device setting as highlighted in this review. Therefore, in selecting an appropriate aerosol generation regime for intense use, the following parameters need to be considered: puff duration, puff volume, puff frequency, puff profile, puff number, battery charge status, coil or atomiser age, voltage setting, ventilation setting, and device orientation. These parameters are not independent 
and the settings should be based on representative human usage rather than the maximum extremes of each individual parameter. Further work to understand how the combination of these parameters affects the amount of aerosol generated will be central to defining protocols for testing and regulating e-cigarettes.

\section{AUTHOR CONTRIBUTIONS}

The manuscript was pulled together by Dr Xavier Cahours and independently reviewed by Dr Krishna Prasad.

\section{ACKNOWLEDGEMENTS}

We would like to express our gratitude to members of CORESTA Product Use Behaviour sub-group who provided their diverse perspective and technical expertise during the drafting of this review.

\section{AUTHORS INFORMATION}

Dr Krishna Prasad is the Co-ordinator and Dr Xavier Cahours is the Secretary of Product Use Behaviour SubGroup of the CORESTA.

\section{REFERENCES}

1. Mangan, D.: E-cigarette Sales Are Smoking Hot, Set to Hit \$1.7 Billion.; CNBC (2013), available at: www. cnbc.com/id/100991511 (accessed August 2018).

2. Euromonitor International: The E-cigarettes Market: Past, Present and Future; 2015 available at: blog.euromonitor.com/2015/06/the-e-cigarettes-marketpast-present-and-future.html (accessed August 2018).

3. Blank, M.D., S. Disharoon, and T. Eissenberg: Comparison of Methods for Measurement of Smoking Behavior: Mouthpiece-Based Computerized Devices Versus Direct Observation; Nicotine Tob. Res. 11 (2009) 896-903. DOI: 10.1093/ntr/ntp083

4. Marian, C., R.J. O’Connor, M.V. Djordjevic, V.W. Rees, D.K. Hatsukami, and P.G. Shields: Reconciling Human Smoking Behavior and Machine Smoking Patterns: Implications for Understanding Smoking Behavior and the Impact on Laboratory Studies; Cancer Epidemiol. Biomarkers Prev. 18 (2009) 3305-3320. DOI: 10.1158/1055-9965.EPI-09-1014

5. International Organization for Standardization (ISO): ISO/TR 17219:2013. Review of Human Smoking Behaviour and Recommendations for a New ISO Standard for the Machine Smoking of Cigarettes; ISO, Geneva, Switzerland, 2013.

6. Vas, C.A., C.Ü. Yurteri, C.J. Dickens, and K. Prasad: Development and Characterisation of a Smoking Behaviour Measurement System; Beitr. Tabakforsch. Int. 26 (2015) 219-231. DOI: 10.1515/cttr-2015-0010

7. Gregg, E., T. Bachmann, R. Bito, X. Cahours, M. McEwan, P. Nelson, K. Prasad, G. Scherer, and M. Stiles: Assessing Smoking Behaviour and Tobacco Smoke Exposure: Definition and Methods; Beitr.
Tabakforsch. Int. 25 (2013) 685-699.

DOI: $10.2478 / \mathrm{cttr}-2013-0945$

8. International Organization for Standardization (ISO): ISO 3308:2012. Routine Analytical Smoking Machine -- Definition and Standard Conditions; ISO, Geneva, Switzerland, 2012.

9. International Organization for Standardization (ISO): ISO 20778. Cigarettes -- Routine Analytical Cigarette Smoking Machine -- Definitions and Standard Conditions with an Intense Smoking Regime; ISO, Geneva, Switzerland, under development.

10. Cooperation Center for Scientific Research Relative to Tobacco (CORESTA) - E-Cigarette Task Force: Technical Report - 2014 Electronic Cigarette Aerosol Parameters Study (published March 2015), available at: www.coresta.org/2014-electronic-cigarette-aerosolparameters-study-29232.html (accessed August 2018).

11. Cooperation Center for Scientific Research Relative to Tobacco (CORESTA): Recommended Method No. 81: Routine Analytical Machine for E-Cigarette Aerosol Generation and Collection -- Definitions and Standard Conditions; 2015, available at: www.coresta.org/rou tine-analytical-machine-e-cigarette-aerosol-generationand-collection-definitions-and-standard (accessed August 2018).

12. Hua, M., H. Yip, and P. Talbot: Mining Data on Usage of Electronic Nicotine Delivery Systems (ENDS) from YouTube Videos; Tob. Control 22 (2013) 103-106. DOI: 10.1136/tobaccocontrol-2011-050226

13. Farsalinos, K.E., G. Romagna, D. Tsiapras, S. Kyrzopoulos, and V. Voudris: Evaluation of Electronic Cigarette Use (Vaping) Topography and Estimation of Liquid Consumption: Implications for Research Protocol Standards Definition and for Public Health Authorities' Regulation; Int. J. Environ. Res. Public Health 10 (2013) 2500-2514. DOI: 10.3390/ijerph10062500.

14. Strasser, A.A., V. Souprountchouk, A. Kaufmann, S. Blazekovic, F. Leone, N.L. Benowitz, and R.A. Schnoll: Nicotine Replacement, Topography, and Smoking Phenotypes of E-Cigarettes; Tob. Regul. Sci. 2 (2016) 352-362. DOI: 10.18001/TRS.2.4.7

15. St Helen, G., K.C. Ross, D.A. Dempsey, C.M. Havel, P. Jacob, and N.L. Benowitz: Nicotine Delivery and Vaping Behavior during Ad Libitum E-Cigarette Access; Tob. Regul. Sci. 2 (2016) 363-376.

DOI: $10.18001 /$ TRS.2.4.8

16. Behar, R.Z., M. Hua, and P. Talbot: Puffing Topography and Nicotine Intake of Electronic Cigarette Users; PloS One 10 (2015) e0117222, 18 pages.

DOI: $10.1371 /$ journal.pone.0117222

17. Norton, K.J., K.M. June, and R.J. O’Connor: Initial Puffing Behaviors and Subjective Responses Differ Between an Electronic Nicotine Delivery System and Traditional Cigarettes; Tob. Induc. Dis. 12 (2014) 17. DOI: 10.1186/1617-9625-12-17

18. Ramôa, C.P., M.M. Hiler, T.R. Spindle, A.A. Lopez, N. Karaoghlanian, T. Lipato, A.B. Breland, A. Shihadeh, and T. Eissenberg: Electronic Cigarette Nicotine Delivery Can Exceed That of Combustible Cigarettes: A Preliminary Report; Tob. Control 25 (2016) e6-e9. DOI: 10.1136/tobaccocontrol-2015-052447

19. Lopez, A.A., M.M. Hiler, E.K. Soule, C.P. Ramôa, 
N.V. Karaoghlanian, T. Lipato, A.B. Breland, A.L. Shihadeh, and T. Eissenberg: Effects of Electronic Cigarette Liquid Nicotine Concentration on Plasma Nicotine and Puff Topography in Tobacco Cigarette Smokers: A Preliminary Report; Nicotine Tob. Res. 18 (2016) 720-723.

20. Cunningham, A., S. Slayford, C.A. Vas, J. Gee, S. Costigan, and K. Prasad: Development and Validation of a Device for Measuring Puffing Topography of E-Cigarette Users; Presentation ST56 at the CORESTA Congress, Quebec, CANADA 2014, available at: https://www.coresta.org/abstracts/development-and-v alidation-device-measuring-puffing-topography-e-cig arette-users-28003 (accessed August 2018)

21. Patwardhan, S.: E-Cigarette Consumption and Puffing Topography Data; Presentation at the FDA CTP Workshop, Hyattsville, ML, USA, 2015.

22. Cunningham, A., S. Slayford, C. Vas, J. Gee, S. Costigan, and K. Prasad: Development, Validation and Application of a Device to Measure E-Cigarette Users' Puffing Topography; Sci. Rep. 6 (2016) 35071. DOI: $10.1038 /$ srep35071

23. Connell, C., D. Bennett, N. Meruva, A. Vansickel, and J. Edmiston: Validation of a Smoking Puff Analyzer for Use with an Electronic Cigarette Prototype; Poster presented at the 68th Tobacco Science Research Conference (TSRC), Charlottesville, VA, USA, 2014.

24. Edmiston, J., Y. Zhao, Q. Liang, A. Vansickel, J. Liu, and M. Sarkar: Characterization of Puff Topography During 8-Hours of Ad Libitum Use of MarkTen ${ }^{\circledR}$ E-Vapor Products; Presentation at the 70th Tobacco Science Research Conference (TSRC), Palm Beach, FL, USA, 2016.

25. Farsalinos, K.E., A. Spyrou, C. Stefopoulos, K. Tsimopoulou, P. Kourkoveli, D. Tsiapras, S. Kyrzopoulos, K. Poulas, and V. Voudris: Nicotine Absorption From Electronic Cigarette Use: Comparison Between Experienced Consumers (Vapers) and Naïve Users (Smokers); Sci. Rep. 5 (2015) 11269, 8 pages. DOI: $10.1038 /$ srep 11269

26. Robinson, R.J., E.C. Hensel, P.N. Morabito, and K.A. Roundtree: Electronic Cigarette Topography in the Natural Environment; PloS One 10 (2015) e0129296, 14 pages. DOI: 10.1371/journal.pone.0129296

27. Robinson, R.J., E.C. Hensel, K.A. Roundtree, A.G. Difrancesco, J.M. Nonnemaker, and Y.O. Lee: Week Long Topography Study of Young Adults Using Electronic Cigarettes in Their Natural Environment; PloS One 11 (2016) e0164038, 31 pages.

DOI: 10.1371 /journal.pone.0164038

28. Lee, Y.O., J.M. Nonnemaker, B. Bradfield, E.C. Hensel, and R.J. Robinson: Examining Daily Electronic Cigarette Puff Topography Among Established and Non-Established Cigarette Smokers in Their Natural Environment; Nicotine Tob. Res. (2017). DOI: $10.1093 / \mathrm{ntr} / \mathrm{nt} \times 222$

29. Dautzenberg, B. and D. Bricard: Real-Time Characterization of E-Cigarettes Use: The 1 Million Puffs Study; J. Addict. Res. Ther. 6 (2015) 229. DOI: $10.4172 / 2155-6105.1000229$

30. Lee, Y.H., M. Gawron, and M.L. Goniewicz: Changes in Puffing Behavior Among Smokers Who Switched
From Tobacco to Electronic Cigarettes; Addict. Behav. 48 (2015) 1-4. DOI: 10.1016/j.addbeh.2015.04.003

31. Hiler, M., A. Breland, T. Spindle, S. Maloney, T. Lipato, N. Karaoghlanian, A. Shihadeh, A. Lopez, C. Ramôa, and T. Eissenberg: Electronic Cigarette User Plasma Nicotine Concentration, Puff Topography, Heart Rate, and Subjective Effects: Influence of Liquid Nicotine Concentration and User Experience; Exp. Clin. Psychopharmacol. 25 (2017) 380-392. DOI: $10.1037 /$ pha0000140

32. Dawkins, L.E., C.F. Kimber, M. Doig, C. Feyerabend, and O. Corcoran: Self-Titration by Experienced E-cigarette Users: Blood Nicotine Delivery and Subjective Effects; Psychopharmacology 233 (2016) 2933-2941. DOI: $10.1007 / \mathrm{s} 00213-016-4338-2$

33. Wagener, T.L., E.L. Floyd, I. Stepanov, L.M. Driskill, S.G. Frank, E. Meier, E.L. Leavens, A.P. Tackett, N. Molina, and L. Queimado: Have Combustible Cigarettes Met Their Match? The Nicotine Delivery Profiles and Harmful Constituent Exposures of Second-Generation and Third-Generation Electronic Cigarette Users; Tob. Control 26 (2017) e23-e28.

DOI: 10.1136/tobaccocontrol-2016-053041

34. Farsalinos, K., K. Poulas, and V. Voudris: Changes in Puffing Topography and Nicotine Consumption Depending on the Power Setting of Electronic Cigarettes; Nicotine Tob. Res. 20 (2018) 993-997.

DOI: $10.1093 / \mathrm{ntr} / \mathrm{ntx} 219$

35. Spindle, T.R., M.M. Hiler, A.B. Breland, N.V. Karaoghlanian, A.L. Shihadeh, and T. Eissenberg: The Influence of a Mouthpiece-Based Topography Measurement Device on Electronic Cigarette User's Plasma Nicotine Concentration, Heart Rate, and Subjective Effects Under Directed and Ad Libitum Use Conditions; Nicotine Tob. Res. 19 (2017) 469-476.

DOI: $10.1093 / \mathrm{ntr} / \mathrm{ntw} 174$

36. Spindle, T.R., A.B. Breland, N.V. Karaoghlanian, A.L. Shihadeh, and T. Eissenberg: Preliminary Results of an Examination of Electronic Cigarette User Puff Topography: The Effect of a Mouthpiece-Based Topography Measurement Device on Plasma Nicotine and Subjective Effects; Nicotine Tob. Res. 17 (2015) 142-149. DOI: $10.1093 / \mathrm{ntr} / \mathrm{ntu} 186$

37. McNeill, A., L.S. Brose, R. Calder, S.C. Hitchman, P. Hajek, and H. McRobbie: E-Cigarettes: An Evidence Update. A Report Commissioned by Public Health England; Public Health England Publications, London, UK, 2015, available at: www.gov.uk/government/uploads/ system/uploads/attachment_data/file/457102/Eciga rettes an evidence update A report_commissioned by_Public_Health_England_FINAL.pdf.

\section{Corresponding author:}

Krishna Prasad

British American Tobacco

Regents Park Road

Southampton, SO15 8TL

United Kingdom

E-mail: krishna_prasad@bat.com 